\title{
Smoking-Induced Oxidative Stress in Bone: The Effects on Bone Turnover
}

\author{
Júlia Benini Kohler', Jader Joel Machado Junqueira', Taysa Cristiane Moreira da Silva ${ }^{3}$, Marco Antonio \\ Gonçalves Pontes Filho ${ }^{3,4}$, lolanda De Fátima L C Tibério', Fernanda Degobbi T Q S Lopes', Alexandre \\ Póvoa Barbosa',2*
}

\author{
'Department of Medicine, Laboratory of Experimental Therapeutics (LIM-20), School of Medicine, University of Sao Paulo, Sao Paulo, Brazil \\ ${ }^{2}$ Department of Orthopaedics, Hospital do Coração - SP Brazil \\ ${ }^{3}$ Department of Rheumatology, School of Medicine, University of Sao Paulo, Sao Paulo, Brazil \\ ${ }^{4}$ Departments of Orthopaedics and Rheumatology, School of Medicine, University of Sao Paulo, Sao Paulo, Brazil
}

\section{Article Info}

\section{Article Notes}

Received: May 14, 2021

Accepted: July 09, 2021

\section{${ }^{*}$ Correspondence:}

${ }^{*}$ Dr. Alexandre Póvoa Barbosa, Departments of Orthopaedics and Rheumatology, School of Medicine, University of Sao Paulo, Sao Paulo, Brazil; Telephone No: 551121652354 Email: ortopediabarbosa@gmail.com

${ }^{\ominus} 2021$ Barbosa AP. This article is distributed under the terms of the Creative Commons Attribution 4.0 International License.

\section{Keywords}

Smoking

Bone loss

Bone turnover

Bone matrix

Oxidative stress

Bone fragility

\section{Abstract}

Despite of clinical evidence of increased incidence of bone diseases amongst smokers as well as worsening recovery in orthopedic surgeries, it is still unclear which pathological mechanisms are induced by smoking and how these events impair bone turnover.

Animal models and in vitro studies have been used to better elucidate these questions and smoking-induced oxidative stress have been pointed as playing crucial role in the worsening of bone cells activities leading bone damage.

Oxidative stress is a physiological mechanism characterized by an imbalance between oxidants and antioxidants components. This imbalance leads cell damage and consequent release of inflammatory mediators, resulting in structural changes that impair the functionality of compromised organ.

In this review, we summarize findings from clinical, animal models and in vitro studies that have elucidated the importance of the oxidative stress induced by smoking in different bone cells activities, leading bone mineral and organic matrix structural changes.

Highlights: We present the newest findings in understanding the impact of smoking in bone matrix composition. Review the clinical and experimental evidence for smoking-induced oxidative stress potential roles in bone turnover. Descript future directions for research and clinical management.

\section{Introduction}

\section{Smoking effects on bone: Epidemiological evidence}

Cigarette smoking is recognized as an important risk factor for several diseases, such as chronic obstructive pulmonary disease, cancer, heart attack and vascular diseases ${ }^{1-4}$.

Smoking is associated with increased incidence of fractures ${ }^{5}$, osteoporosis, mainly in women ${ }^{6}$, and delayed consolidation under bone injury conditions ${ }^{7}$.

In clinical studies, smoking has been associated with decreases in bone volume and mineral density (BMD) by the enhancement of bone resorption. Moreover, these effects depend on the duration of smoking and body weight $t^{6,8,9}$.

As the volume of exposure to cigarettes increase (expressed by cigarettes per day or pack-years) higher will be the decline in BMD at multiple skeletal sites and bone mass ${ }^{10}$.

The impacts of smoking on bone may differ between men and 
women. Since there are few studies describing these differences, there was no consensus about the physiological mechanisms that leads these different responses ${ }^{6}$.

The deleterious effect of smoking has been mostly demonstrated in women with low levels of estrogen, and in these women it was observed a reduction in the response of hormonal replacement therapy ${ }^{11,12}$. The United States Department of Health and Human Services showed that smoking causes a decrease in BMD in menopausal women ${ }^{13}$, and this effect is progressive over the years in smokers compared with nonsmokers ${ }^{14}$.

Although it is well established the importance of second-hand smoking in the progression of many diseases and health conditions, including cancer ${ }^{15}$, cardiovascular diseases ${ }^{16}$ and respiratory diseases ${ }^{17}$; little is known about the influence of passive smoking on bone metabolism. Only a few studies have investigated how passive smoking could induce negative effects on bone mass and fracture risk ${ }^{18-}$ 21. In contrast, extensive data from epidemiological and experimental studies indicate that gene-environmental interaction during pregnancy and early life leads permanent changes in physiological homeostasis, increasing many diseases predisposition by epigenetic mechanisms ${ }^{22}$. Exposure to tobacco smoke, at the early events during pregnancy and childhood, could promote deleterious effects on the development process and may result in permanent damage $\mathrm{e}^{23-27}$.

Despite the evidence supporting the deleterious effects of smoking on bone health in both genders, it is still unclear how smoking impairs bone turnover and reduces bone mass ${ }^{28,29}$.

Tobacco smoke contains gaseous and particular compounds that induce free radical release which may lead to chronic airway inflammation and tissue destruction ${ }^{30-32}$. Evidence from studies in dental surgeries have strongly attributed the deleterious effects of smoking as result of an increase in oxidative stress mechanism ${ }^{33,34}$.

In trauma surgeries, it has been showed that the reactive organic radicals contained in cigarette smoke induce delayed healing of fractures as well as high risk of nonunion after fracture through cell toxicity and inflammatory process induction ${ }^{35,36,37}$.

Oxidative stress is a physiological mechanism characterized by an imbalance between oxidants and antioxidants components. This imbalance leads cell damage and consequent release of inflammatory mediators, resulting in structural changes that impair the functionality of compromised $\operatorname{organ}^{33}$.

In this review we summarize some results from clinical, animal models and in vitro studies (Figure 2). that have highlighted the importance of the oxidative stress induced by smoking in the worsening of bone turnover, focusing in bone mineral and organic matrix structural changes.

\section{Increased Reactive Oxygen Species production: Oxidative Stress}

Oxidative stress is a physiological mechanism characterized by an imbalance between oxidants and antioxidants components. This imbalance leads to cell damage and consequent release of inflammatory mediators, resulting in structural changes that impair the functionality of compromised organ (Figure 1$)^{38}$.

Enzymatic reactions such as those involved in respiratory chain, cytochrome P450 system, phagocytosis, and prostaglandin synthesis are recognized by oxidants generation $^{39-41}$. Reactive oxygen species (ROS) are oxygencontaining molecules and free radicals components recognized by their oxidant activity. Among them, Hydroxyl $(\mathrm{OH}-)$, superoxide radicals (O2 -), hydrogen peroxide $\left(\mathrm{H}_{2} \mathrm{O}_{2}\right)$, singlet oxygen, and lipid peroxides are the most described. They are composed by one or more unpaired electrons, which makes them highly reactive since they find out another electron to fill their orbital and stabilize their electron balance. These metabolites show little halflife and are difficult to measure in biological samples from humans, but it is possible to measure their deleterious effects in proteins, lipids, and DNA ${ }^{38}$.

02- is mainly produced during cellular respiration by mitochondria, also by action of lipoxygenases (LOX) and cyclooxygenases (COX) during the arachidonic acid metabolism and by endothelial and inflammatory cells ${ }^{6}$. Once formed, 02 - will be involved in several reactions that could generate hydroxyl $(\mathrm{OH}-)$, hydrogen peroxide $\left(\mathrm{H}_{2} \mathrm{O}_{2}\right)$ and peroxynitrite (ONOO-). Hydroxyl radical $(\mathrm{OH} \bullet)$ is the most reactive among all the free radical species and is delivered by Fenton reaction: 02 - - with $\mathrm{H} 2 \mathrm{O} 2$, with $\mathrm{Fe} 2+$ or $\mathrm{Cu}+$ as a reaction catalyst $\mathrm{t}^{42-44}$. Nitric oxide radical (NO)is synthesized from arginine-to-citrulline oxidation by nitric oxide synthase (NOS) $)^{42-44}$.

In addition to endogenous sources, ROS could be generated from exogenous sources. Exposure to environmental pollutants, heavy metals, chemical solvents, cigarette smoke and others that must be degraded or metabolized could induce the production of free radicals ${ }^{45}$. The maintenance of the exposure to these sources leads the development and progression of different diseases such cancer, chronic obstructive pulmonary diseases, osteoporosis $^{38}$.

When there was an imbalance between ROS formation and the capability of cells to remove them, the oxidative stress mechanism will occur, affecting cellular structures, such as membranes, proteins, lipids and desoxyribonucleic acid (DNA $)^{46}$. An excess of hydroxyl radical and peroxynitrite culminates in lipid peroxidation which damages cell membranes and lipoproteins. In proteins, oxidative stress changes their chemical structures which worsens 


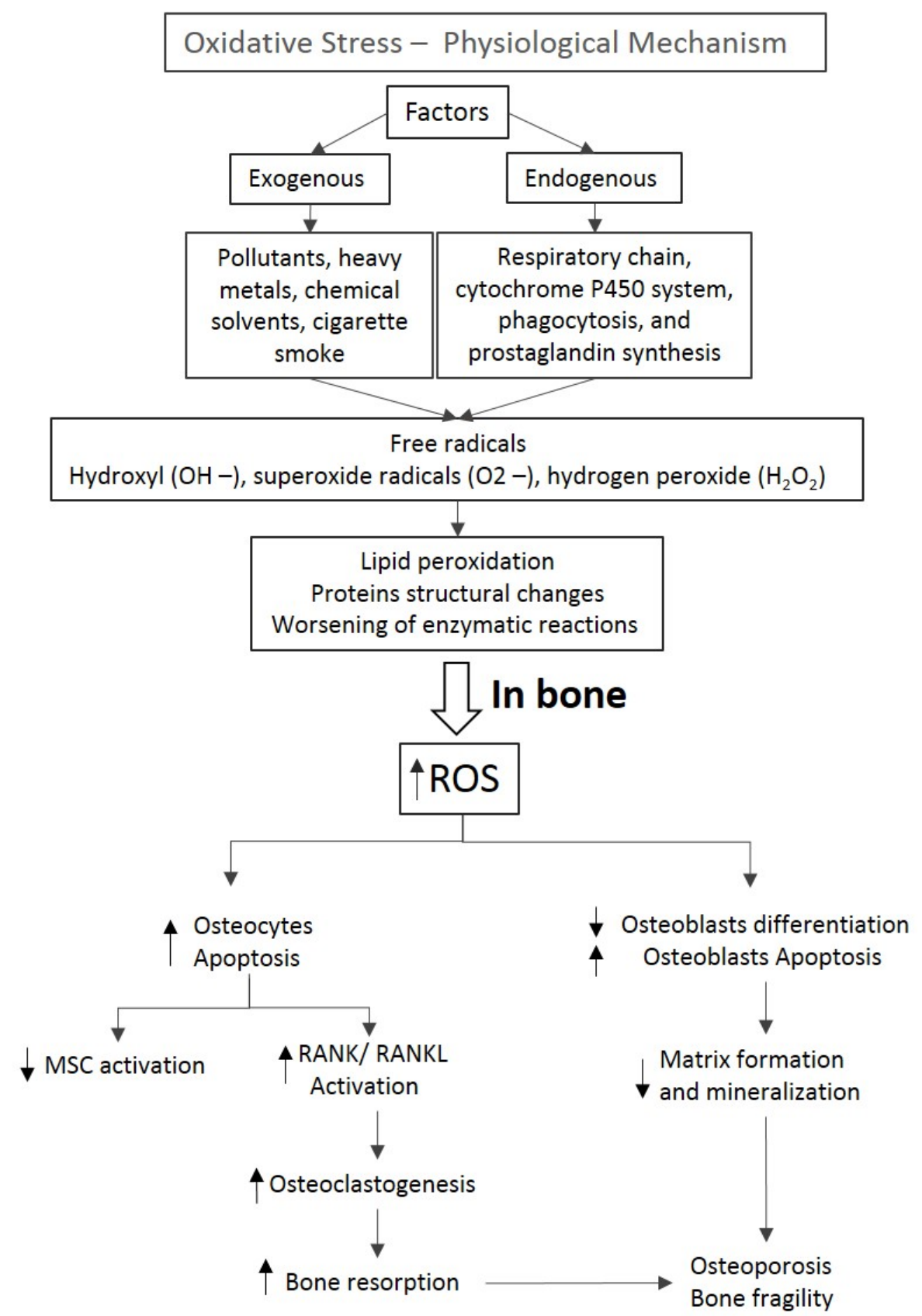

Figure 1: Oxidative stress is a physiological mechanism that depends on the exogenous and endogenous factors which could lead to an increase of free radicals. These metabolites induce damages of cell membranes and lipoproteins, compromising cells activity. In bone, the increase of ROS leads to an increase in osteocytes and osteoblasts apoptosis as well as a decrease in osteoblasts differentiation. Moreover, the increase in RANK/RANKL activation induces osteoclastogenesis and a sequent bone resorption. These events culminate in changes in organic and mineral matrix, compromising the bone integrity. (ROS: Reactive Oxygen Species; RANK: Receptor Activator of Nuclear Factor k B; RANKL: Receptor Activator of Nuclear Factor kB ligand; MSCs Mesenchymal stromal cells)

their enzymatic activity. In DNA, the damages induced by oxidative stress leads mutagenesis and also compromises the epigenetic formations ${ }^{38}$.

To avoid the effects of oxidative stress, cells establish a defensive system based mainly on the production enzymas with antioxidant actions, such as superoxide dismutase (SOD), catalase (CAT), and glutathione peroxidase $(\mathrm{GPx})^{47}$.
However, during extensive exposure to exogenous factors such as cigarette smoke, the cells antioxidant capacity is insufficient to avoid the oxidative stress occurrence. Evidence have been attesting that smoking is associated with increase in ROS production since smokers have lower antioxidant enzyme levels and it impacts in bone resorption and bone mass loss ${ }^{29}$. 


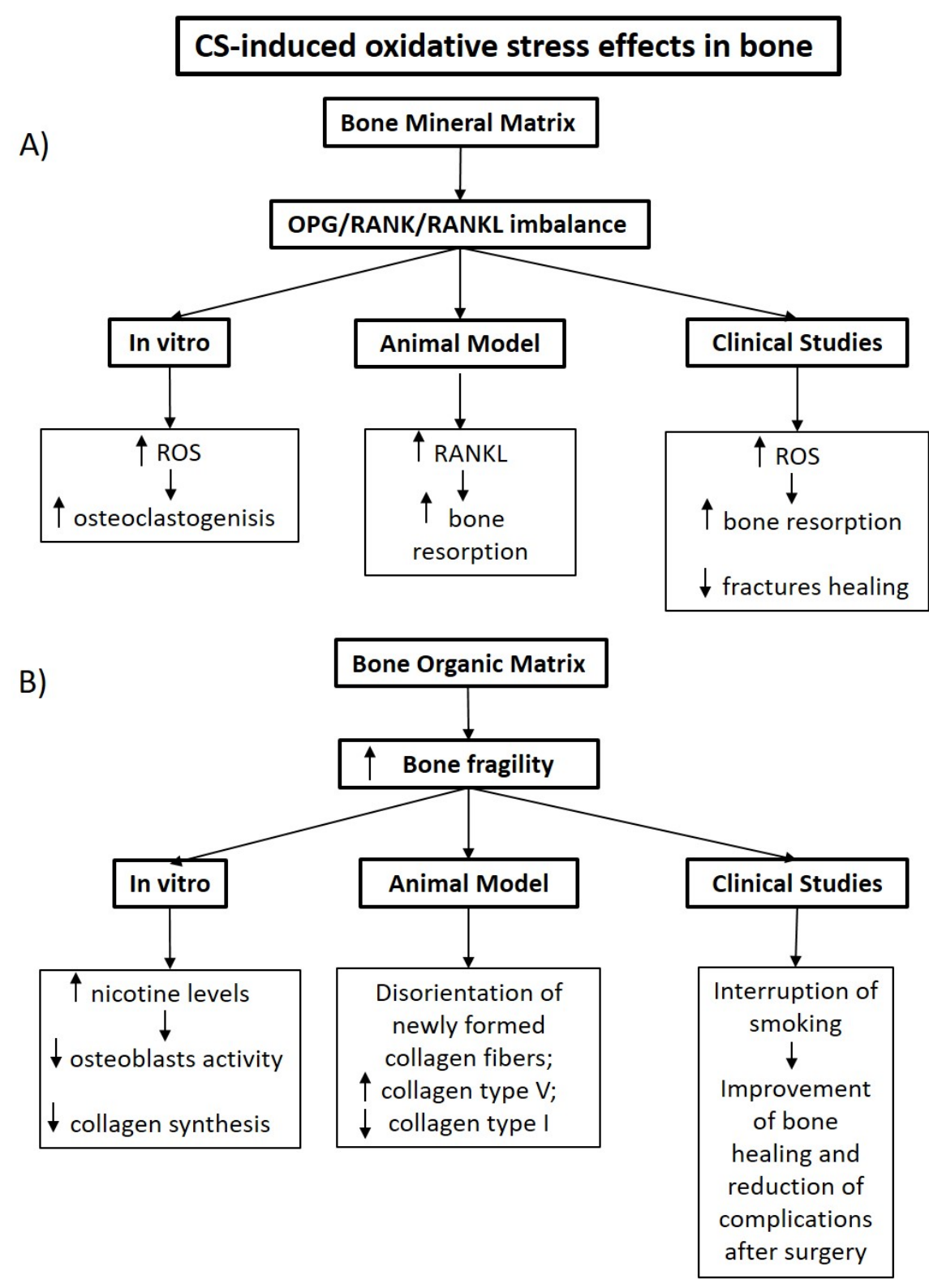

Figure 2: The effects of the CS-induced oxidative stress showed in experimental and clinical studies in bone mineral (A) and organic (B) matrix. (ROS: Reactive Oxygen Species; RANKL: Receptor Activator of Nuclear Factor kB ligand)

The smoking-induced oxidative stress effects in bone turn over

\section{Oxidative stress effects in bone mineral matrix}

Inorganic and organic components are present in bone matrix. Although the inorganic constituent of bone matrix is mainly composed by crystalline hydroxyapatite[Ca3(PO4)2] $3 \mathrm{Ca}(\mathrm{OH}) 2$, the organic component contains around 20 proteins, and the type I collagen is the most prevalent ${ }^{48}$. During the bone resorption, the dissolution of crystalline hydroxyapatite occurs earlier than the injury of the organic components ${ }^{49}$.

Since bone formation requires the recruitment, proliferation and osteogenic differentiation of mesenchymal progenitors cells, the balance between cell activation, cellular activity and cell life span can produce an effect in bone tissue formation and bone density.

The osteocytes, cells in close contact with blood capillaries are considered the major regulators of bone remodeling. The increase in oxidative stress leads to these cells apoptosis which are related to altered bone formation and lower mineral density ${ }^{50,51}$. Also, osteocyte apoptosis is associated with the increased expression of osteoclastogenic factors and elevation in bone turnover, resulting in bone mass $\operatorname{loss}^{52}$. Moreover, the presence of ROS damages stem cells self-renewal activation and differentiation towards 
tissure-specific lineages like osteoblasts and osteocytes ${ }^{53}$. Bone turnover is mainly performed by the osteoclasts and osteoblasts. The activation and proliferation of these cells are regulated by different factors, such as the RANK-RANKLOPG system, growth factors, cytokines and hormones ${ }^{54}$. Osteoclasts are large multinuclear cells and are the only cells capable of resorbing the mineralized bone matrix ${ }^{55}$. The osteoclasts are derived from hematopoietic stem cells (HSCs), which differentiate into bone-resorbing osteoclasts through a series of steps leading to matrix disruption ${ }^{56}$. The maturation of HSCs into osteoclasts is driven forward by several crucial steps and is highly dependent on the presence of Receptor Activator for Nuclear Factor $\kappa \mathrm{B}$ (RANK), the cytokine macrophage stimulating factor (MCS-F) as well as the regulator osteoprotegerin (OPG) ${ }^{57}$.

Osteoblasts are cells derived from mesenchymal progenitors in the bone marrow. They are accumulated in larger groups producing the extracellular matrix components, which is initially unmineralized ${ }^{58}$.

The bone turnover is a complex process and the effectiveness of signaling pathway OPG/RANK/RANKL prevents the bone loss ${ }^{59}$. RANKL is expressed by osteoblasts, osteocytes and other mesenchymal lineage cells ${ }^{60}$. RANKL by binding to its receptor RANK expressed by osteoclasts induces this cell type differentiation and activation. OPG is a circulating receptor that binds to RANKL, inhibiting the osteoclastogenesis, and are present in bone and vascular endothelial cells ${ }^{61,62}$.

The deficiency of RANKL was described associated with severe osteoporosis in mice and decreased osteoclasts activity in in vitro studies ${ }^{63,64}$. In smokers, the imbalance of OPG/RANK/RANKL signaling pathways was described associated with decreased bone mineral density (BMD) and higher susceptibility to bone fractures ${ }^{65,66}$ and some studies have attributed these effects to an increase in reactive oxygen species.

The RANK activation by RANKL induces an increase in reactive oxygen species (ROS) release ${ }^{67,68}$, and the excessive activity of osteoclasts has been described in bone diseases such as osteoporosis ${ }^{67,69,70}$.

In a periodontitis mice model, Kubota et al. $^{71}$ demonstrated that the systemic administration of cigarette smoke condensate or nicotine accelerated the alveolar bone destruction through an increased expression of RANKL by osteoclasts.

In this context, Kim et al..$^{70}$ showed in experimental and in vitro studies that an antioxidant protein capable of destroying $\operatorname{ROS}^{72-79}$, negatively regulated RANKL stimulated signals and reduced the osteoclastogenesis in bone, under normal and injury conditions.

Corroborating with these data, some findings have attested the antioxidant effects by using a resveratrol and resveratrol-like compounds, resulting in downregulation in osteoclastogenesis and osteoclast activity in periodontal diseases, revealing this therapeutic approach as promissor in improving the bone healing processes even under smoking conditions ${ }^{80,81}$.

Also, increased levels of oxidants, such as isoprostane (iso-PGF2 $\alpha$ ) in urine and plasma samples have been demonstrated in association with reduced bone mineral density $^{82,83}$. These results highlighted possible markers to monitor the increased oxidative stress occurrence associated with reduced bone mineral density as well the possibility of treatments with antioxidants to control the osteoporosis progression.

\section{Oxidative stress effects in bone organic matrix}

The organic components of bone tissue include collagen fiber types I and V, proteoglycans, growth factors and cytokines. Type I collagen represents approximately $90 \%$ of the total collagen forming the protein scaffold upon which mineral is deposited ${ }^{84}$. The pattern of collagen fibers distribution in mature bone is characterized by dense parallel layers that alternate between parallel and orthogonal distribution ${ }^{85-87}$.

Type I collagen are not only the most abundant component in organic matrix, but also contributes to tissue organization due its mechanical properties and fibril distribution, that it is essential for mineralization process $^{86,87}$. In this context, the damage of type I collagen synthesis has dramatic effects on the skeleton ${ }^{88}$.

Sasaki et al. demonstrate that long-term CS exposure in mice impairs the normal growth of lumbar vertebral bodies and deteriorates vertebral bone quality, and these results were attributed mainly to disorientation of newly total collagen fibers that were replaced during remodeling process after the CS-induced injury in bone tissue ${ }^{89}$.

Other important collagen subtype in bone turnover is the collagen type V. Although, it counts for $<3 \%$ of total collagen, this is a regulatory fibril-forming collagen since regulates the fibrillogenesis of the collagen types I and III. In a previous study, we showed in mice, that cigarette smoke exposure alters bone matrix composition and impairs bone mineralization resulting in bone fragility. It occurs due to increased production and deposition of collagen $\mathrm{V}$ with a concomitant decrease of collagen type $\mathrm{I}^{84}$.

The organic components of bone matrix are mainly produced by the osteoblasts and these cells activity could be compromised by excessive oxidative stress. Thus, oxidative stress has been recognized as important factor to impair bone formation in postmenopausal and senile osteoporosis $^{90,91}$. 
Zhang et al. ${ }^{92}$ showed in transgenic mice that deficiency in Fox01, a protein that modulate the expression of genes involved in cell differentiation, DNA damage repair, and oxidative stress, induced a decrease in osteoblasts differentiation and an increase in caspase-3 activity, resulting in these cells apoptosis. Authors demonstrated that Fox01 provides a favorable intracellular environment for osteoblast functions by defensing against the adverse effects of oxidative stress.

To better elucidate the CS-induced effects in osteoblasts, the most used approach is to test the different levels of nicotine in in vitro studies. The increase in oxidative stress induced by nicotine has been demonstrated in osteoblasts, worsening this cell function ${ }^{6}$. Moreover, these effects seem to be dose-dependent, at high concentrations of nicotine, osteoblast-like cells demonstrate decreased proliferation and impaired collagen synthesis ${ }^{93}$, whereas at low levels these cells are stimulated ${ }^{34}$.

It is important to note that different ways of nicotine administration could interfere in its systemic absorption, altering bone turnover impact. The inhalation of nicotine induces higher levels of this substance compared with subcutaneously administration. Thus, clinical studies have been showed that the interruption of smoking habit before the elective surgeries diminishes the time required for bone healing as well the complications after surgery ${ }^{94}$. Lower levels of nicotine and cotinine, that is a compound of nicotine, are detected in serum and urine of patients after smoking cessation ${ }^{95}$. Probably, part of these benefits could be attributed to the decreased systemic levels of nicotine and a consequent oxidative stress reduction after a smoking cessation.

\section{Clinical aspects of smoking and bone health}

Osteoporosis is characterized by low BMD and weakening of bone tissue, which leads to gradual bone fragility and can causes fractures. Among all the treatable causes of osteoporosis, smoking has been established as an important contributing risk factor. It affects the balance of the naturally occurring processes of bone resorption and bone formation, resulting in low BMD as the amount resorbed is not totally replaced ${ }^{29}$.

Few studies are available regarding the pathophysiological mechanisms by which smoking would lead to bone loss and the assessment of the clinical association of them. Smoking is thought to cause low bone density through numerous pathways.

Among the currently available studies, there is the fact that, in general, smokers have lower weight and body mass index (BMI), which is postulated to provide an osteogenic stimulus and it is linked to higher $\mathrm{BMD}^{6,29}$. They also found lower serum levels of vitamin D in smokers, which is required for good bone health. Furthermore, there is an impaired intestinal calcium absorption associated with changes in the metabolism of calciotropic hormones. Smoking has been linked to changes in hormone household, leading to a decrease in parathyroid hormone (i.e., responsible for the reducing calcium absorption) and low estrogen levels, as well as to a state of hypercortisolism and high levels of adrenal androgens $s^{6,8,29,96}$. Those changes have been linked to an increased risk of osteoporosis. It is imperative to reassure that woman who smoke have alterations in the metabolism of sex hormones leading to a lower level of estradiol and menopause at an earlier age when compared to non-smokers ${ }^{96}$. When men are evaluated, the studies are conflicting, but some have shown inhibition of aromatase (similar to that found in women) and, consequently, reduced production of estradiol from testosterone. In addition, there is a negative impact on the angiogenesis required for bone metabolism ${ }^{29}$.

Smokers are more likely to suffer from peripheral vascular disease, reducing the bone blood supply. As smokers are weaker with less lean mass, they have poorer balance and impaired neuromuscular performance, which may also increase the risk of falls ${ }^{29}$.

Few studies are available regarding the effect of smoking cessation on bone health. One study found an intermediate risk of fracture in ex-smokers. And another one discovered that the effect of smoking on bone density was reversible, and the bone density of ex-smokers improved in less than 10 years (i.e., may be partially reversible), and there is a dose-response relationship for the amount in pack-years smoked and fracture risk. Remarkably, other studies reported that the effects of smoking cessation in postmenopausal women produced improvement in gonadal hormones, level of bone formation, and resorption markers and improvement in the bone density after cessation/reduction ${ }^{29,97,98}$.

Another two studies reported that subjects exposed to secondhand smoke had significantly lower phalangeal BMD and higher risk for femoral neck osteoporosis than unexposed subjects ${ }^{29,97,98}$.

OS is generated as a result of insufficient activity of the endogenous antioxidant defense system against reactive oxygen species (ROS) ${ }^{99}$. The results have been further shown by cross-sectional and case-control studies, in which OS was characterized by a high level of F2-isoprostanes in urine and a low level of antioxidant enzymes in blood, along with a reduced bone mineral density and an increased risk of osteoporosis ${ }^{100,101}$. Studies suggested that homocysteine (Hcy) played an important role in bone metabolism and had been involved in osteoporotic facture incidence ${ }^{102-104}$. The results of a meta-analysis showed increased Hcy and nitric oxide (NO) in the postmenopausal osteoporosis (PO) subjects, while it showed decreased levels of folate and total antioxidant power (TAP), along with lower 
activity of superoxide dismutase (SOD) and glutathione peroxidase $(\mathrm{GPx})$ in these subjects. The imbalance of ROS and antioxidant system may contribute to functional and structural remodeling that favors the occurrence of $\mathrm{PO}^{99}$.

\section{Final considerations and new directions}

Despite of clinical evidence of increased incidence of bone diseases amongst smokers as well as worsening recovery in orthopedic surgeries, it is still unclear which pathological mechanisms are induced by CS and how these events impair bone turnover.

To describe these pathological mechanisms, animal models and in vitro studies have been performed to describe different bone cells activities and signaling pathways regulating cells interactions. Part of these findings pointed out the CS-induced oxidative stress importance in the impairment of bone turnover resulting in damage of mineral and organic bone matrix which corroborate with clinical studies that have extensively showed lower BMD and increased bone fragility in smokers.

Although it is difficult to perform measurement of ROS due to its short half-life, there are some clinical studies that showed increased levels of F2 isoprostanes as well as 8-isoprostanes in urine samples form smokers compared with non-smokers ${ }^{105}$ and in patients with osteoporosis, revealing the oxidative stress occurrence. However, until now, the majority of experimental and clinical studies have evaluated levels of vitamins, or antioxidant enzymes, which are informative; but they only reflect one side of the redox homeostasis, leaving the question of whether decreased levels are actually indicative of increased oxidative stress occurrence ${ }^{99}$. In this context, the administration of antioxidant compounds showed a reduction in ROS production in experimental studies and beneficial results in smokers with periodontal diseases, revealing this therapeutic approach as promisor to ameliorate bone healing even under smoking conditions.

Clinical management are still mainly based in radiographic images. X-Rays and CT-Scans reveal only the final result of bone structural changes. It is not possible to investigate the different cell types and not even the components of bone matrix separately. Moreover, the majority of these exams detect structural changes only after the establishment of bone diseases that are worsening by smoking.

The association between radiographic images and specific biomarkers in serum could be useful to facilitate the clinical management. These measurements could be performed in tissue as well in serum or urine samples to evaluate the impact of smoking cessation in the diminishment of oxidative stress markers and how it impacts bone turnover, revealing the best moment for elective orthopedic surgeries. Also, it will be useful to evaluate the efficacy of different treatments, such as antioxidants, in different bone diseases that are worsening by smoking.

\section{Conflict of Interest}

The authors declare no conflicts of interest.

\section{References}

1. Erhardt L. Cigarette smoking: an undertreated risk factor for cardiovascular disease. Atherosclerosis. 2009; 205(1): 23-32.

2. Brusselle GG, Joos GF, Bracke KR. New insights into the immunology of chronic obstructive pulmonary disease. Lancet. 2011; 378(9795): 1015-26.

3. Stämpfli MR, Anderson GP. How cigarette smoke skews immune responses to promote infection, lung disease and cancer. Nat Rev Immunol. 2009; 9(5): 377-84.

4. Global Initiative for Chronic Obstructive Lung Disease. Global strategy for the diagnosis, management, and prevention of chronic obstructive pulmonary disease 2019 report. 2019.

5. Vestergaard P, Mosekilde L. Fracture risk associated with smoking: a meta-analysis. Journal of internal medicine. 2003; 254(6): 572-83.

6. Yoon V, Maalouf NM, Sakhaee K. The effects of smoking on bone metabolism. Osteoporos Int. 2012; 23(8): 2081-92.

7. Bender D J-KT, Biglari B, Swing T, et al. Cigarette smoking and its impact on fracture healing. Trauma. 2014.

8. Bjarnason $\mathrm{NH}$, Christiansen $\mathrm{C}$. The influence of thinness and smoking on bone loss and response to hormone replacement therapy in early postmenopausal women. The Journal of clinical endocrinology and metabolism. 2000; 85(2): 590-6.

9. Tamaki J, Iki M, Fujita Y, et al. Impact of smoking on bone mineral density and bone metabolism in elderly men: the Fujiwara-kyo Osteoporosis Risk in Men (FORMEN) study. Osteoporos Int. 2011; 22(1): 133-41.

10. Ward KD, Klesges RC. A meta-analysis of the effects of cigarette smoking on bone mineral density. Calcified tissue international. 2001; 68(5): 259-70.

11. Daniel M, Martin AD, Drinkwater DT. Cigarette smoking, steroid hormones, and bone mineral density in young women. Calcified tissue international. 1992; 50(4): 300-5.

12. Kapoor D, Jones TH. Smoking and hormones in health and endocrine disorders. European journal of endocrinology. 2005; 152(4): 491-9.

13. The Health Consequences of Smoking: A Report of the Surgeon General. Reports of the Surgeon General. Atlanta (GA). 2004.

14. Law MR, Hackshaw AK. A meta-analysis of cigarette smoking, bone mineral density and risk of hip fracture: recognition of a major effect. Bmj. 1997; 315(7112): 841-6.

15. IARC International agency for reasearch on cancer. World Health Organization L. Tobacco smoke and involuntary smoking, IARC. 2002.

16. Barnoya J, Glantz SA. Cardiovascular effects of secondhand smoke: nearly as large as smoking. Circulation. 2005; 111(20): 2684-98.

17. Chan-Yeung M, Dimich-Ward H. Respiratory health effects of exposure to environmental tobacco smoke. Respirology. 2003; 8(2): 131-9.

18. Blum M, Harris SS, Must A, et al. Household tobacco smoke exposure is negatively associated with premenopausal bone mass. Osteoporos Int. $2002 ; 13(8): 663-8$.

19. Afghani A, Xie B, Wiswell RA, et al. Bone mass of asian adolescents in China: influence of physical activity and smoking. Medicine and science in sports and exercise. 2003; 35(5): 720-9. 
20. Altunbayrak O, Saridogan M, Ateser B, et al. Passive smoking and postmenopausal osteoporosis. Bone. 2009.

21. Hsu YH BM, Venners SA, Terwedow H, et al. Second-hand smoking increases the risks of osteoporosis and osteoporotic fractures in Chinese men and women. 7th International Symposium on Osteoporosis. 2006.

22. Waterland RA, Michels KB. Epigenetic epidemiology of the developmental origins hypothesis. Annual review of nutrition. 2007; 27: 363-88.

23. Braun M, Klingelhöfer D, Oremek GM, et al. Influence of SecondHand Smoke and Prenatal Tobacco Smoke Exposure on Biomarkers, Genetics and Physiological Processes in Children-An Overview in Research Insights of the Last Few Years. Int J Environ Res Public Health. 2020; 17(9): 3212.

24. von Ehrenstein OS, von Mutius E, Maier E, et al. Lung function of school children with low levels of alpha1-antitrypsin and tobacco smoke exposure. The European respiratory journal. 2002; 19(6): 1099-106.

25. Gilliland FD, Li YF, Dubeau L, et al. Effects of glutathione S-transferase M1, maternal smoking during pregnancy, and environmental tobacco smoke on asthma and wheezing in children. Am J Respir Crit Care Med. 2002; 166(4): 457-63.

26. Kabesch M, Hoefler C, Carr D, et al. Glutathione S transferase deficiency and passive smoking increase childhood asthma. Thorax. 2004; 59(7): 569-73.

27. Chen Y, Wong GW, Li J. Environmental Exposure and Genetic Predisposition as Risk Factors for Asthma in China. Allergy, asthma \& immunology research. 2016; 8(2): 92-100.

28. Cusano NE. Skeletal Effects of Smoking. Current osteoporosis reports. 2015; 13(5): 302-9.

29. Al-Bashaireh AM, Haddad LG, Weaver M, et al. The Effect of Tobacco Smoking on Bone Mass: An Overview of Pathophysiologic Mechanisms. Journal of osteoporosis. 2018; 2018: 1206235.

30. Hsieh MM, Everhart JE, Byrd-Holt DD, et al. Prevalence of neutropenia in the U.S. population: age, sex, smoking status, and ethnic differences. Annals of internal medicine. 2007; 146(7): 486-92.

31. Wallace AM, Sandford AJ, English JC, et al. Matrix metalloproteinase expression by human alveolar macrophages in relation to emphysema. Copd. 2008; 5(1): 13-23.

32. Ito JT, Lourenco JD, Righetti RF, et al. Extracellular Matrix Component Remodeling in Respiratory Diseases: What Has Been Found in Clinical and Experimental Studies? Cells. 2019; 8(4): 342.

33. van der Vaart H, Postma DS, Timens W, et al. Acute effects of cigarette smoke on inflammation and oxidative stress: a review. Thorax. 2004; 59(8): 713-21.

34. Carnevali S, Petruzzelli S, Longoni B, et al. Cigarette smoke extract induces oxidative stress and apoptosis in human lung fibroblasts. Am J Physiol Lung Cell Mol Physiol. 2003; 284(6): L955-63.

35. Chen F, Osterman AL, Mahony K. Smoking and bony union after ulnashortening osteotomy. American journal of orthopedics. 2001; 30(6): 486-9.

36. Little CP, Burston BJ, Hopkinson-Woolley J, et al. Failure of surgery for scaphoid non-union is associated with smoking. Journal of hand surgery. 2006; 31(3): 252-5.

37. Javed F, Rahman I, Romanos GE. Tobacco-product usage as a risk factor for dental implants. Periodontol 2000. 2019; 81(1): 48-56.

38. Pizzino G, Irrera N, Cucinotta M, et al. Oxidative Stress: Harms and Benefits for Human Health. Oxidative medicine and cellular longevity 2017; 2017: 8416763.

39. B. Halliwell and J. M. C. Gutteridge. Free Radicals in Biology and Medicine. Clarendon Press, Oxford, UK, 4th edition, 2007.
40. T. Bahorun MAS, V. Luximon-Ramma, O. I. Aruoma. Free radicals and antioxidants in cardiovascular health and disease. Internet Journal of Medical Update. 2006; 1: pp 1-17.

41. P. Pacher JSB, L. Liaudet. Nitric oxide and peroxynitrite in health and disease Physiological Reviews. 2007; 87: 315-424.

42. S. Kumar and A. K. Pandey. Free radicals: health implications and their mitigation by herbals. British Journal of Medicine and Medical Research. 2015; 7: 438-57.

43. Kumar S, Pandey AK. Chemistry and biological activities of flavonoids: an overview. The Scientific World Journal. 2013.

44. Willcox JK, Ash SL, Catignani GL. Antioxidants and prevention of chronic disease. Critical reviews in food science and nutrition. 2004; 44(4): 275-95.

45. Valko M, Morris H, Cronin MT. Metals, toxicity and oxidative stress. Current medicinal chemistry. 2005; 12(10): 1161-208.

46. Genestra M. Oxyl radicals, redox-sensitive signalling cascades and antioxidants. Cellular signalling. 2007; 19(9): 1807-19.

47. Deponte M. Glutathione catalysis and the reaction mechanisms of glutathione-dependent enzymes. Biochimica et biophysica acta. 2013; 1830(5): 3217-66.

48. Boskey AL, Posner AS. Bone structure, composition, and mineralization. The Orthopedic clinics of North America. 1984; 15(4): 597-612.

49. Blair HC, Kahn AJ, Crouch EC, et al. Isolated osteoclasts resorb the organic and inorganic components of bone. The Journal of cell biology. 1986; 102(4): 1164-72.

50. Bellido T. Osteocyte-driven bone remodeling. Calcified tissue international. 2014; 94(1): 25-34.

51. Bonewald LF. The amazing osteocyte. J Bone Miner Res. 2011; 26(2): 229-38.

52. Domazetovic V, Marcucci G, Iantomasi T, et al. Oxidative stress in bone remodeling: role of antioxidants. Clin Cases Miner Bone Metab. 2017; 14(2): 209-16.

53. Chen JR, Lazarenko OP, Zhang J, et al. Diet-derived phenolic acids regulate osteoblast and adipocyte lineage commitment and differentiation in young mice. J Bone Miner Res. 2014; 29(5): 104353.

54. Vega D, Maalouf NM, Sakhaee K. CLINICAL Review \#: the role of receptor activator of nuclear factor-kappaB (RANK)/RANK ligand/ osteoprotegerin: clinical implications. J Clin Endocrinol Metab. 2007; 92(12): 4514-21.

55. Roodman GD. Cell biology of the osteoclast. Experimental hematology. 1999; 27(8): 1229-41.

56. Roodman GD. Regulation of osteoclast differentiation. Annals of the New York Academy of Sciences. 2006; 1068: 100-9.

57. Wiktor-Jedrzejczak W, Bartocci A, Ferrante AW, et al. Total absence of colony-stimulating factor 1 in the macrophage-deficient osteopetrotic (op/op) mouse. Proceedings of the National Academy of Sciences of the United States of America. 1990; 87(12): 4828-32.

58. Brylka LJ, Schinke T. Chemokines in Physiological and Pathological Bone Remodeling. Frontiers in immunology. 2019; 10: 2182

59. Atkins GJ, Welldon KJ, Holding CA, et al. The induction of a catabolic phenotype in human primary osteoblasts and osteocytes by polyethylene particles. Biomaterials. 2009; 30(22): 3672-81.

60. Burguet A, Kaminski M, Abraham-Lerat L, et al. The complex relationship between smoking in pregnancy and very preterm delivery. Results of the Epipage study. BJOG: an international journal of obstetrics and gynaecology. 2004; 111(3): 258-65. 
61. Eghbali-Fatourechi G, Khosla S, Sanyal A, et al. Role of RANK ligand in mediating increased bone resorption in early postmenopausal women. The Journal of clinical investigation. 2003; 111(8): 1221-30.

62. Demir B, Haberal A, Geyik P, et al. Identification of the risk factors for osteoporosis among postmenopausal women. Maturitas. 2008; 60(34): 253-6.

63. Fuller $\mathrm{K}$, Wong B, Fox $\mathrm{S}$, et al. TRANCE is necessary and sufficient for osteoblast-mediated activation of bone resorption in osteoclasts. The Journal of experimental medicine. 1998; 188(5): 997-1001.

64. Kong YY, Yoshida H, Sarosi I, et al. OPGL is a key regulator of osteoclastogenesis, lymphocyte development and lymph-node organogenesis. Nature. 1999; 397(6717): 315-23.

65. Wang XF, Zhang YK, Yu ZS, et al. The role of the serum RANKL/OPG ratio in the healing of intertrochanteric fractures in elderly patients. Molecular medicine reports. 2013; 7(4): 1169-72.

66. LaCroix AZ, Jackson RD, Aragaki A, et al. OPG and sRANKL serum levels and incident hip fracture in postmenopausal Caucasian women in the Women's Health Initiative Observational Study. Bone. 2013; 56(2): 474-81.

67. Lee NK, Choi YG, Baik JY, et al. A crucial role for reactive oxygen species in RANKL-induced osteoclast differentiation. Blood. 2005; 106(3): 852-9.

68. Ha H, Kwak HB, Lee SW, et al. Reactive oxygen species mediate RANK signaling in osteoclasts. Experimental cell research. 2004; 301(2): 119-27.

69. Steinbeck MJ, Kim JK, Trudeau MJ, et al. Involvement of hydrogen peroxide in the differentiation of clonal HD-11EM cells into osteoclastlike cells. Journal of cellular physiology. 1998; 176(3): 574-87.

70. Kim HS, Nam ST, Mun SH, et al. DJ-1 controls bone homeostasis through the regulation of osteoclast differentiation. Nature communications. 2017; 8(1): 1519.

71. Kubota M, Yanagita M, Mori K, et al. The Effects of Cigarette Smoke Condensate and Nicotine on Periodontal Tissue in a Periodontitis Model Mouse. PloS one. 2016; 11(5): e0155594.

72. Kim DK, Kim HS, Kim AR, et al. DJ-1 regulates mast cell activation and IgE-mediated allergic responses. The Journal of allergy and clinical immunology. 2013; 131(6): 1653-62.

73. Kim JM, Jang HJ, Choi SY, et al. DJ-1 contributes to adipogenesis and obesity-induced inflammation. Scientific reports. 2014; 4: 4805.

74. Lev N, Barhum Y, Lotan I, et al. DJ-1 knockout augments disease severity and shortens survival in a mouse model of ALS. PloS one. 2015; 10(3): e0117190.

75. Won KJ, Jung SH, Jung SH, et al. DJ-1/park7 modulates vasorelaxation and blood pressure via epigenetic modification of endothelial nitric oxide synthase. Cardiovascular research. 2014; 101(3): 473-81.

76. Xu XM, Moller SG. ROS removal by DJ-1: Arabidopsis as a new model to understand Parkinson's Disease. Plant signaling \& behavior. 2010; 5(8): 1034-6.

77. Kinumi T, Kimata J, Taira T, et al. Cysteine- 106 of DJ-1 is the most sensitive cysteine residue to hydrogen peroxide-mediated oxidation in vivo in human umbilical vein endothelial cells. Biochemical and biophysical research communications. 2004; 317(3): 722-8.

78. Taira T, Saito Y, Niki T, et al. DJ-1 has a role in antioxidative stress to prevent cell death. EMBO reports. 2004; 5(2): 213-8.

79. Cuevas S, Zhang Y, Yang Y, et al. Role of renal DJ-1 in the pathogenesis of hypertension associated with increased reactive oxygen species production. Hypertension. 2012; 59(2): 446-52.

80. Murgia D, Mauceri R, Campisi G, et al. Advance on Resveratrol Application in Bone Regeneration: Progress and Perspectives for Use in Oral and Maxillofacial Surgery. Biomolecules. 2019; 9(3): 94.
81. Correa MG, Absy S, Tenenbaum H, et al. Resveratrol attenuates oxidative stress during experimental periodontitis in rats exposed to cigarette smoke inhalation. Journal of periodontal research. 2019; 54(3): 225-32.

82. Lee YJ, Hong JY, Kim SC, et al. The association between oxidative stress and bone mineral density according to menopausal status of Korean women. Obstetrics \& gynecology science. 2015; 58(1): 46-52.

83. Ostman B, Michaelsson K, Helmersson J, et al. Oxidative stress and bone mineral density in elderly men: antioxidant activity of alphatocopherol. Free radical biology \& medicine. 2009; 47(5): 668-73.

84. Barbosa AP, Lourenco JD, Junqueira JJM, et al. The deleterious effects of smoking in bone mineralization and fibrillar matrix composition. Life sciences. 2020; 241: 117132.

85. Murshed M, Harmey D, Millan JL, et al. Unique coexpression in osteoblasts of broadly expressed genes accounts for the spatial restriction of ECM mineralization to bone. Genes \& development. 2005; 19(9): 1093-104.

86. Ricard-Blum S. The collagen family. Cold Spring Harbor perspectives in biology. 2011; 3(1): a004978.

87. Morello R. Osteogenesis imperfecta and therapeutics. Matrix biology: journal of the International Society for Matrix Biology. 2018; 71-72: 294-312.

88. Sasaki M, Chubachi S, Kameyama N, et al. Effects of long-term cigarette smoke exposure on bone metabolism, structure, and quality in a mouse model of emphysema. PloS one. 2018; 13(1): e0191611.

89. Narisawa S, Yadav MC, Millan JL. In vivo overexpression of tissuenonspecific alkaline phosphatase increases skeletal mineralization and affects the phosphorylation status of osteopontin. J Bone Miner Res. 2013; 28(7): 1587-98.

90. Knuutinen A, Kokkonen N, Risteli J, et al. Smoking affects collagen synthesis and extracellular matrix turnover in human skin. The British journal of dermatology. 2002; 146(4): 588-94.

91. Sorensen LT, Zillmer R, Agren M, et al. Effect of smoking, abstention, and nicotine patch on epidermal healing and collagenase in skin transudate. Wound Repair Regen. 2009; 17(3): 347-53.

92. Zhang Y, Xiong Y, Zhou J, et al. Fox01 expression in osteoblasts modulates bone formation through resistance to oxidative stress in mice. Biochemical and biophysical research communications. 2018; 503(3): 1401-8.

93. Lee JJ, Patel R, Biermann JS, et al. The musculoskeletal effects of cigarette smoking. The Journal of bone and joint surgery American volume. 2013; 95(9): 850-9.

94. Carlson BB, Burton DC, Jackson RS, et al. Recidivism Rates After Smoking Cessation Before Spinal Fusion. Orthopedics. 2016; 39(2): e318-22.

95. Wong PK, Christie JJ, Wark JD. The effects of smoking on bone health. Clinical science. 2007; 113(5): 233-41.

96. Kim KH, Lee CM, Park SM, et al. Secondhand smoke exposure and osteoporosis in never-smoking postmenopausal women: the Fourth Korea National Health and Nutrition Examination Survey. Osteoporos Int. 2013; 24(2): 523-32.

97. Ko CH, Chan RL, Siu WS, et al. Deteriorating effect on bone metabolism and microstructure by passive cigarette smoking through dual actions on osteoblast and osteoclast. Calcified tissue international. 2015; 96(5): 389-400.

98. Ajiro Y, Tokuhashi Y, Matsuzaki H, et al. Impact of passive smoking on the bones of rats. Orthopedics. 2010; 33(2): 90-5.

99. Zhou Q, Zhu L, Zhang D, et al. Oxidative Stress-Related Biomarkers in Postmenopausal Osteoporosis: A Systematic Review and MetaAnalyses. Disease markers. 2016; 2016: 7067984. 
100. Sanchez-Rodriguez MA, Ruiz-Ramos M, Correa-Munoz E, et al Oxidative stress as a risk factor for osteoporosis in elderly Mexicans as characterized by antioxidant enzymes. BMC musculoskeletal disorders. 2007; 8: 124

101. Basu S, Michaelsson K, Olofsson H, et al. Association between oxidative stress and bone mineral density. Biochemical and biophysical research communications. 2001; 288(1): 275-9.

102. Leboff MS, Narweker R, LaCroix A, et al. Homocysteine levels and risk of hip fracture in postmenopausal women. The Journal of clinical endocrinology and metabolism. 2009; 94(4): 1207-13.
103. McLean RR, Jacques PF, Selhub J, et al. Homocysteine as a predictive factor for hip fracture in older persons. The New England journal of medicine. 2004; 350(20): 2042-9.

104. van Meurs JB, Dhonukshe-Rutten RA, Pluijm SM, et al. Homocysteine levels and the risk of osteoporotic fracture. The New England journal of medicine. 2004; 350(20): 2033-41.

105. Khan NA, Lawyer G, McDonough S, et al. Systemic biomarkers of inflammation, oxidative stress and tissue injury and repair among waterpipe, cigarette and dual tobacco smokers. Tobacco control. 2020; 29(Suppl 2): s102-s9. 